\title{
CONTRACTIVITY AND GROUND STATE DOMINATION PROPERTIES FOR NON-LOCAL SCHRÖDINGER OPERATORS
}

\author{
KAMIL KALETA, MATEUSZ KWAŚNICKI, JÓZSEF LŐRINCZI
}

\begin{abstract}
We study supercontractivity and hypercontractivity of Markov semigroups obtained via ground state transformation of non-local Schrödinger operators based on generators of symmetric jump-paring Lévy processes with Kato-class confining potentials. This class of processes has the property that the intensity of single large jumps dominates the intensity of all multiple large jumps, and the related operators include pseudo-differential operators of interest in mathematical physics. We refine these contractivity properties by the concept of $L^{p}$-ground state domination and its asymptotic version, and derive sharp necessary and sufficient conditions for their validity in terms of the behaviour of the Lévy density and the potential at infinity. As a consequence, we obtain for a large subclass of confining potentials that, on the one hand, supercontractivity and ultracontractivity, on the other hand, hypercontractivity and asymptotic ultracontractivity of the transformed semigroup are equivalent properties. This is in stark contrast to classical Schrödinger operators, for which all these properties are known to be different.
\end{abstract}

\section{INTRODUCTION}

In recent years Markov processes with jumps and non-local pseudo-differential operators, which are generators of these processes, have received much attention in both pure and applied mathematics. They allow to model a variety of discontinuous random phenomena in statistical mechanics, anomalous transport, laser optics and, via a Feynman-Kac-type representation, relativistic quantum theory and quantum models with spin. The non-local character often poses intriguing problems, especially in areas where functional analysis, PDE and probability theory meet.

In many interesting cases the specific models involve non-local Schrödinger operators based on generators of Lévy processes with jumps. Recent investigations include heat trace and spectral gap estimates [1, 5, 21], gradient estimates of harmonic functions [31], properties of radial solutions, ground states, eigenfunctions and eigenvalues [32, 33, 23, 13, 26, 35, 18], smoothing properties of evolution semigroups [25, 9, 22, properties of the associated transformed jump processes [24, 28], as well as applications in quantum theory [34, 17, 16, 19, 15, 3].

In this paper we focus on fundamental properties of semigroups $\left\{T_{t}: t \geq 0\right\}$ of non-local Schrödinger operators of the form $H=-L+V$ by using functional integral techniques. Here $L$ is the generator of a symmetric jump Lévy process in $\mathbf{R}^{d}, d \geq 1$, with the property that all multiple large jumps are dominated under the Lévy measure with density $\nu$ by a single large jump (which we call a jump-paring Lévy process). This property proves to be a unifying concept including many jump processes and related operators of interest [25]. The term $V$ will be chosen to be a Kato-class confining potential (see Section 3.1 below for precise definitions), thus the operator $H$ has a purely discrete spectrum, consisting of eigenvalues $\lambda_{0}<\lambda_{1} \leq \lambda_{2} \leq \ldots \rightarrow \infty$ of finite

Key-words: symmetric Lévy process, non-local Schrödinger operator, Feynman-Kac semigroup, ground state transformation, $L^{p}$-ground state domination, intrinsic hypercontractivity, intrinsic supercontractivity, (asymptotic) intrinsic ultracontractivity

2010 MS Classification: Primary 47D08, 60G51; Secondary 47D03, 47G20

Research supported by the National Science Center (Poland) grant on the basis of the decision No. DEC2011/03/D/ST1/00311 and by the Foundation for Polish Science. 
multiplicities. The unique eigenfunction $\varphi_{0}$ corresponding to eigenvalue $\lambda_{0}$, called ground state, has a continuous, bounded and strictly positive version (in Lebesgue sense), which will be our choice throughout below.

We define the ground state-transformed (also known as intrinsic) semigroup $\left\{\widetilde{T}_{t}: t \geq 0\right\}$ associated with the non-local Schrödinger operator $H$ by

$$
\widetilde{T}_{t} f(x)=\frac{e^{\lambda_{0} t}}{\varphi_{0}(x)} T_{t}\left(f \varphi_{0}\right)(x), \quad f \in L^{2}\left(\mathbf{R}^{d}, \mu\right), \quad t \geq 0,
$$

where $\mu(d x)=\varphi_{0}^{2}(x) d x$. The semigroup $\left\{\widetilde{T}_{t}: t \geq 0\right\}$ is conservative and determines a right Markov process with stationary distribution $\mu$, which we call ground state-transformed jump process corresponding to $H$ [28, 24].

The aim of this paper is to study the contractivity properties of the operators $\widetilde{T}_{t}$ from $L^{2}\left(\mathbf{R}^{d}, \mu\right)$ to $L^{p}\left(\mathbf{R}^{d}, \mu\right)$ for $p \in(2, \infty)$. Recall that the semigroup $\left\{\widetilde{T}_{t}: t \geq 0\right\}$ is called hypercontractive (resp. supercontractive) if $\widetilde{T}_{t}$ maps $L^{2}\left(\mathbf{R}^{d}, \mu\right)$ into $L^{p}\left(\mathbf{R}^{d}, \mu\right)$ for every $p \in(2, \infty)$ and for some $t>0$ (resp. for every $t>0$ ). Moreover, $\left\{\widetilde{T}_{t}: t \geq 0\right\}$ is ultracontractive (resp. asymptotically ultracontractive) if the same holds with $p=\infty$ for every $t>0$ (resp. for some $t>0$ ). Such smoothing properties have been widely studied for classical Schrödinger operators $H=-\Delta+V$ [41. Hypercontractivity has been introduced by Nelson [38], and proved to be useful especially in quantum field theory. The first works on ultracontractivity are due to Davies and Simon [11], and Bañuelos [4], and it has a number of useful consequences (for a detailed discussion see [25, Sect. 1]). Generally, such contractivity properties are useful in establishing functional (such as log-Sobolev, Nash, Poincaré etc) inequalities, as well as heat kernel estimates [2, 10]. For the classical case there is a natural hierarchy of these properties. For instance, in the case of $H=-\Delta+|x|^{\alpha}\left(\log (1+|x|)^{\beta}\right.$, the related transformed semigroup is not hypercontractive if $\alpha<2$, it is hypercontractive but not supercontractive if $\alpha=2, \beta=0$, it is supercontractive but not ultracontractive if $\alpha=2,0<\beta \leq 2$, and it is ultracontractive if $\alpha=2, \beta>2$, or if $\alpha>2$. This shows that ultracontractivity is essentially stronger than supercontractivity, which is essentially stronger than hypercontractivity. Note that the asymptotic version of ultracontractivity has not been studied in the classical case before the paper [24], where it was proposed first.

For non-local Schrödinger operators the situation is less explored (see [9]), with the exception of ultracontractivity properties, i.e., when $p=\infty$. This has been studied in the recent paper [25] for the same class of underlying jump-paring processes and Kato-class potentials (see this reference also for a discussion of previous literature). An interesting feature of non-local Schrödinger operators is that all eigenfunctions are pointwise dominated everywhere by the ground state [25, Cor. 2.1, Ex. 4.8(5)]. This property occurs even if ultracontractivity of $\left\{\tilde{T}_{t}: t \geq 0\right\}$ fails to hold, which makes a striking difference with the classical case. As it will be seen below, essential differences between classical and non-local Schrödinger operators also occur in respect of their hyper- and supercontractivity properties.

The main results of the present paper are as follows. We first work in a general setting with $L^{2}$ semigroups associated with self-adjoint operators $H$ bounded from below, which satisfy some mild regularity assumptions (Section 2). In this framework we introduce the concept of $L^{p}$-ground state domination of the semigroup $\left\{T_{t}: t \geq 0\right\}$ and its asymptotic version (Definition2.1). By using them, we refine the notions of hypercontractivity and supercontractivity of the transformed semigroup $\left\{\widetilde{T}_{t}: t \geq 0\right\}$ (Theorems 2.1] 2.2), and derive necessary and sufficient conditions for these properties to hold (Proposition 3.2, Theorem 3.1, Corollary 3.1 and Theorem 3.2). These conditions are expressed explicitly in terms of the behaviour of the Lévy density $\nu$ and the potential $V$ at infinity. Surprisingly, 
they are in match with the conditions under which ultracontractivity and its asymptotic version hold, recently obtained in [25, and leads to unexpected equivalences in the smoothing properties of semigroups of non-local Schrödinger operators. For the class of jump-paring Lévy processes and for a large class of confining potentials, we obtain that supercontractivity and hypercontractivity of the transformed semigroup $\left\{\widetilde{T}_{t}: t \geq 0\right\}$ coincide respectively with ultracontractivity and asymptotic ultracontractivity of the semigroup (Corollaries 3.2 3.3. $)$. This has not been observed before, and it makes a stark contrast to classical Schrödinger operators featuring the Laplacian [11, p.396].

Notation. We will use the notation $C_{i}(a, b, \ldots), i=1,2, \ldots$, for positive constants dependent on parameters $a, b, \ldots$ throughout this paper, while dependence on the process $\left(X_{t}\right)_{t \geq 0}$ and on the dimension $d$ is assumed without being explicitly indicated. Auxiliary constants in the proofs are denoted by $c_{i}$. We will also use the notation $f \asymp g$ meaning that there exists a constant $C$ such that $C^{-1} g \leq f \leq C g$ holds. We denote by $\mathbf{1}(x)$ the indicator function of $\mathbf{R}^{d}$.

\section{2. $L^{p}$-GROUND STATE DOMINATION PROPERTIES}

Throughout this section we assume that $H$ is a self-adjoint operator on $L^{2}\left(\mathbf{R}^{d}, d x\right)$ and bounded from below, such that

(a) $\lambda_{0}:=\inf \operatorname{Spec} H$ is a non-degenerate eigenvalue, i.e., a ground state of $H$ exists

(b) $e^{-t H}$ is positivity improving for every $t>0$, i.e., $e^{-t H} f(x)>0$ for all $x \in \mathbf{R}^{d}$ and $f \in$ $L^{2}\left(\mathbf{R}^{d}, d x\right)$ such that $f \geq 0$ and $f \neq 0$ a.e.

(c) $e^{-t H}$ is bounded on $L^{\infty}\left(\mathbf{R}^{d}, d x\right)$ for all $t>0$, and satisfies

$$
\left|e^{-t H} f(x)\right| \leq e^{-t H} \mathbf{1}(x)\|f\|_{\infty}, \quad \text { for a. e. } x \in \mathbf{R}^{d} \text { and every } f \in L^{\infty}\left(\mathbf{R}^{d}, d x\right)
$$

(d) there exists $t_{\mathrm{b}}>0$ such that $e^{-t_{\mathrm{b}} H}$ is bounded from $L^{2}\left(\mathbf{R}^{d}, d x\right)$ to $L^{\infty}\left(\mathbf{R}^{d}, d x\right)$.

The corresponding unique $L^{2}$-normalized eigenfunction $\varphi_{0}$, called ground state, can be assumed to be strictly positive [39, Th. XIII.43], [36, Sect. 3.4.3]. Moreover, it immediately follows from the eigenvalue equation $e^{-t_{\mathrm{b}} H} \varphi_{0}=e^{-\lambda_{0} t_{\mathrm{b}}} \varphi_{0}$ and $(\mathrm{d})$ that $\varphi_{0} \in L^{\infty}\left(\mathbf{R}^{d}, d x\right)$.

The following properties of the semigroup $\left\{e^{-t H}: t \geq 0\right\}$ will be central to our investigations. Denote by $L^{p}\left(\mathbf{R}^{d}, \mu\right), p \in[1, \infty)$, the space of $L^{p}$-integrable functions on $\mathbf{R}^{d}$ weighted by the probability measure $\mu(d x)=\varphi_{0}^{2}(x) d x$. Clearly, we have the identification $L^{\infty}\left(\mathbf{R}^{d}, \mu\right)=L^{\infty}\left(\mathbf{R}^{d}, d x\right)$, and for every $1 \leq q<p \leq \infty$ the inclusions $L^{p}\left(\mathbf{R}^{d}, \mu\right) \subset L^{q}\left(\mathbf{R}^{d}, \mu\right)$ hold.

Definition 2.1 (Ground state domination properties). Let $p \in(2, \infty]$. We say that

(i) the operator $e^{-t H}$ is $L^{p}$-ground state dominated (abbreviated as $L^{p}-G S D$ ) if

$$
\frac{e^{-t H} \mathbf{1}}{\varphi_{0}} \in L^{p}\left(\mathbf{R}^{d}, \mu\right)
$$

(ii) the semigroup $\left\{e^{-t H}: t \geq 0\right\}$ is $L^{p}$-ground state dominated (abbreviated as $L^{p}$-GSD) if for every $t>0$ the operators $e^{-t H}$ are $L^{p}$-ground state dominated

(iii) the semigroup $\left\{e^{-t H}: t \geq 0\right\}$ is asymptotically $L^{p}$-ground state dominated (abbreviated as $\left.L^{p}-A G S D\right)$ if there exists $t_{p}>0$ such that for every $t>t_{p}$ the operators $e^{-t H}$ are $L^{p}$-ground state dominated. If the specific value of $t_{p}$ is essential, we write $\left(t_{p}, L^{p}\right)-A G S D$ to emphasize this.

For $p=\infty$ such a domination property has been recently introduced and used in [25]. The definition above considers this now for all $p \in(2, \infty]$. The $L^{p}$-GSD property expresses (in terms of appropriate weighted $L^{p}$-spaces) the balance between the fall-off of the mass of the semigroup and the decay of the ground state at infinity. The ground state $\varphi_{0}$ is a key concept in many applications in quantum 
and statistical physics (see, e.g., [27]), thus obtaining further "multi-scale" information according to the $L^{p}$-norms on its regularity and localization properties, which is our goal in this paper, is clearly of interest.

We define the ground state-transformed semigroup $\left\{e^{-t \widetilde{H}}: t \geq 0\right\}$ by

$$
e^{-t \widetilde{H}} f(x)=\frac{e^{\lambda_{0} t}}{\varphi_{0}(x)} e^{-t H}\left(f \varphi_{0}\right)(x), \quad f \in L^{2}\left(\mathbf{R}^{d}, \mu\right), \quad t \geq 0 .
$$

The following observations will be fundamental in what follows.

Lemma 2.1. Let $p \in(2, \infty)$. Consider the following two conditions.

(1) For some $t>0$ the operator $e^{-t H}$ is $L^{p}-G S D$.

(2) For some $t>0$ the operator $e^{-t \widetilde{H}}$ is bounded from $L^{2}\left(\mathbf{R}^{d}, \mu\right)$ to $L^{p}\left(\mathbf{R}^{d}, \mu\right)$.

Then we have the following:

(i) If (1) holds for some $t=s>0$, then (2) follows for $t=s+t_{\mathrm{b}}$, where $t_{\mathrm{b}}$ is given by (d).

(ii) If (2) holds for some $t=s>0$ and

$$
\varphi_{0}^{1-\frac{1}{p-1}} \in L^{1}\left(\mathbf{R}^{d}, d x\right),
$$

then (1) follows for $t=2 s$.

Proof. We first prove (i). We have for all $f \in L^{2}\left(\mathbf{R}^{d}, \mu\right)$,

$$
\int_{\mathbf{R}^{d}}\left|e^{-\left(t+t_{\mathrm{b}}\right) \widetilde{H}} f(x)\right|^{p} \mu(d x)=\int_{\mathbf{R}^{d}}\left(\frac{e^{\lambda_{0}\left(t+t_{\mathrm{b}}\right)}}{\varphi_{0}(x)}\left|e^{-t H} e^{-t_{\mathrm{b}} H}\left(f \varphi_{0}\right)(x)\right|\right)^{p} \mu(d x) .
$$

By the standing assumptions (c) and (d), we have

$$
\left|e^{-t H} e^{-t_{\mathrm{b}} H}\left(f \varphi_{0}\right)(x)\right| \leq e^{-t H} \mathbf{1}(x)\left\|e^{-t_{\mathrm{b}} H}\left(f \varphi_{0}\right)\right\|_{\infty} \leq e^{-t H} \mathbf{1}(x)\left\|e^{-t_{\mathrm{b}} H}\right\|_{2, \infty}\|f\|_{L^{2}\left(\mathbf{R}^{d}, \mu\right)},
$$

for almost all $x \in \mathbf{R}^{d}$. Thus

$$
\left(\int_{\mathbf{R}^{d}}\left|e^{-\left(t+t_{\mathrm{b}}\right) \widetilde{H}} f(x)\right|^{p} \mu(d x)\right)^{1 / p} \leq\left\|e^{-t_{\mathrm{b}} H}\right\|_{2, \infty} e^{\lambda_{0}\left(t+t_{\mathrm{b}}\right)}\left(\int_{\mathbf{R}^{d}}\left|\frac{e^{-t H} \mathbf{1}(x)}{\varphi_{0}(x)}\right|^{p} \mu(d x)\right)^{1 / p}\|f\|_{L^{2}\left(\mathbf{R}^{d}, \mu\right)},
$$

which completes the proof of (i).

To show (ii), choose $q$ such that $1 / p+1 / q=1$. By the symmetry of the operator $e^{-t \widetilde{H}}$ and the duality of $L^{p}\left(\mathbf{R}^{d}, \mu\right)$ and $L^{q}\left(\mathbf{R}^{d}, \mu\right)$, boundedness of $e^{-t \widetilde{H}}$ from $L^{2}\left(\mathbf{R}^{d}, \mu\right)$ to $L^{p}\left(\mathbf{R}^{d}, \mu\right)$ implies its boundedness $L^{q}\left(\mathbf{R}^{d}, \mu\right)$ to $L^{2}\left(\mathbf{R}^{d}, \mu\right)$ with the same norm. By (2.2) we have $f:=\frac{1}{\varphi_{0}} \in L^{q}\left(\mathbf{R}^{d}, \mu\right)$, and thus $g_{t}:=e^{-t \widetilde{H}} f \in L^{2}\left(\mathbf{R}^{d}, \mu\right)$. With this we have

$$
\begin{aligned}
\left(\int_{\mathbf{R}^{d}}\left|\frac{e^{-2 t H} \mathbf{1}(x)}{\varphi_{0}(x)}\right|^{p} \mu(d x)\right)^{1 / p} & =e^{-2 \lambda_{0} t}\left(\int_{\mathbf{R}^{d}}\left|e^{-t \widetilde{H}} g_{t}(x)\right|^{p} \mu(d x)\right)^{1 / p} \leq C_{2, p, t} e^{-2 \lambda_{0} t}\left\|g_{t}\right\|_{L^{2}\left(\mathbf{R}^{d}, \mu\right)} \\
& \leq C_{2, p, t}^{2} e^{-2 \lambda_{0} t}\|f\|_{L^{q}\left(\mathbf{R}^{d}, \mu\right)}=C_{2, p, t}^{2} e^{-2 \lambda_{0} t} \int_{\mathbf{R}^{d}} \varphi_{0}^{1-\frac{1}{p-1}}(x) d x<\infty
\end{aligned}
$$

where $C_{2, p, t}$ is the norm of the operator $e^{-t \widetilde{H}}$ from $L^{2}\left(\mathbf{R}^{d}, \mu\right)$ to $L^{p}\left(\mathbf{R}^{d}, \mu\right)$.

\section{Remark 2.1.}

(1) An implication of (ii) in Lemma 2.1 is that whenever

$$
\text { there exists } \delta \in(0,1) \text { such that } \varphi_{0}^{1-\delta} \in L^{1}\left(\mathbf{R}^{d}, d x\right) \text {, }
$$

and $p \geq 1+1 / \delta$, then (2) implies (1) for appropriate $t$. 
(2) Condition (2.3) (or (2.2)) appears to be non-standard. However, often (and in most cases of direct interest, see e.g. [7, 25, 8]) they hold for both classical and non-local Schrödinger operators. In particular, (2.3) holds for all the examples discussed in [25, Sect. 4].

(3) It is straightforward to check that $L^{p}$-GSD implies

$$
\varphi_{0} \in L^{1}\left(\mathbf{R}^{d}, d x\right) .
$$

In this light, it is reasonable to ask whether the assumption (2.3) in Lemma 2.1 (ii) could be relaxed. It does not seem to be immediate to settle if the same is true under the weaker condition (2.4) in the generality of the present framework. In Section 3 below we will show that for a wide selection of non-local Schrödinger operators with confining potentials for which (2.4) automatically holds, the restriction (2.3) can be lifted. This improvement will be based on the observation that assuming (2.4) instead of (2.2) and by following through the argument in the proof of Lemma 2.1 (ii) with $f:=1 / \varphi_{0}^{1 / q}$, we have

$$
\frac{e^{-2 t H} \varphi_{0}^{1 / p}}{\varphi_{0}} \in L^{p}\left(\mathbf{R}^{d}, \mu\right)
$$

This property is much weaker than (2.1), but still can be applied well whenever strong enough estimates of $\varphi_{0}$ at infinity are available.

In what follows, the above $L^{p}$-ground state domination properties will be applied to characterize the hypercontractivity and supercontractivity properties of transformed semigroups corresponding to non-local Schrödinger operators. Recall the following terminology from 11 .

\section{Definition 2.2 (Contractivity properties).}

(i) The semigroup $\left\{e^{-t \widetilde{H}}: t \geq 0\right\}$ is supercontractive if for every $p \in(2, \infty)$ and $t>0$ the operators $e^{-t \widetilde{H}}$ are bounded from $L^{2}\left(\mathbf{R}^{d}, \mu\right)$ to $L^{p}\left(\mathbf{R}^{d}, \mu\right)$.

(ii) The semigroup $\left\{e^{-t H}: t \geq 0\right\}$ is called intrinsically supercontractive (abbreviated as ISC) if the semigroup $\left\{e^{-t \widetilde{H}}: t \geq 0\right\}$ is supercontractive.

(iii) The semigroup $\left\{e^{-t \widetilde{H}}: t \geq 0\right\}$ is hypercontractive if for every $p \in(2, \infty)$ there exists $t_{p}>0$ such that for every $t \geq t_{p}$ the operators $e^{-t \widetilde{H}}$ are bounded from $L^{2}\left(\mathbf{R}^{d}, \mu\right)$ to $L^{p}\left(\mathbf{R}^{d}, \mu\right)$.

(iv) The semigroup $\left\{e^{-t H}: t \geq 0\right\}$ is called intrinsically hypercontractive (abbreviated as IHC) if the semigroup $\left\{e^{-t \widetilde{H}}: t \geq 0\right\}$ is hypercontractive.

The next two theorems are the main results of this section, providing a necessary and sufficient condition for ISC and IHC in terms of the $L^{p}$-GSD and $L^{p}$-AGSD properties. This will allow us to refine, study and interpret strong smoothness properties of the semigroups $\left\{e^{-t \widetilde{H}}: t \geq 0\right\}$ through the fall-off properties of the mass of $e^{-t H}$, i.e., the decay of the functions $e^{-t H} \mathbf{1}(x)$ when $|x| \rightarrow \infty$.

Theorem 2.1. (IHC and $L^{p}$-AGSD) The following hold:

(i) If the semigroup $\left\{e^{-t H}: t \geq 0\right\}$ is IHC and there exists $\delta \in(0,1)$ such that $\varphi_{0}^{1-\delta} \in$ $L^{1}\left(\mathbf{R}^{d}, d x\right)$, then for every $p \in(2, \infty)$ the semigroup $\left\{e^{-t H}: t \geq 0\right\}$ is $L^{p}-A G S D$. Specifically, if for every $p \in(2, \infty)$ there exists $t_{p}>0$ such that for all $t \geq t_{p}$ the operators $e^{-t \widetilde{H}}$ are bounded from $L^{2}\left(\mathbf{R}^{d}, \mu\right)$ to $L^{p}\left(\mathbf{R}^{d}, \mu\right)$, then for every $p \in(2, \infty)$ the semigroup $\left\{e^{-t H}: t \geq 0\right\}$ is $\left(2\left(t_{p} \vee t_{p_{\delta}}\right), L^{p}\right)-A G S D$, where $p_{\delta}=1+1 / \delta$.

(ii) If for every $p \in(2, \infty)$ the semigroup $\left\{e^{-t H}: t \geq 0\right\}$ is $L^{p}-A G S D$, then the semigroup $\left\{e^{-t H}: t \geq 0\right\}$ is IHC. Specifically, if for every $p \in(2, \infty)$ there exists $t_{p}>0$ such that for all $t \geq t_{p}$ the semigroup $\left\{e^{-t H}: t \geq 0\right\}$ is $\left(t_{p}, L^{p}\right)-A G S D$, then for every $p \in(2, \infty)$ and all $t \geq t_{\mathrm{b}}+t_{p}$ the operators $e^{-t \widetilde{H}}$ are bounded from $L^{2}\left(\mathbf{R}^{d}, \mu\right)$ to $L^{p}\left(\mathbf{R}^{d}, \mu\right)$. 
Proof. This is obtained by a direct application of Lemma 2.1 (and Remark 2.1 (1)) and the monotonicity in $p \in(2, \infty)$ of the inclusions of the $L^{p}\left(\mathbf{R}^{d}, \mu\right)$ spaces.

Theorem 2.2. (ISC and $L^{p}$-GSD) The following hold:

(i) If the semigroup $\left\{e^{-t H}: t \geq 0\right\}$ is ISC and there exists $\delta \in(0,1)$ such that $\varphi_{0}^{1-\delta} \in$ $L^{1}\left(\mathbf{R}^{d}, d x\right)$, then for every $p \in(2, \infty)$ the semigroup $\left\{e^{-t H}: t \geq 0\right\}$ is $L^{p}-G S D$.

(ii) If for every $p \in(2, \infty)$ the semigroup $\left\{e^{-t H}: t \geq 0\right\}$ is $L^{p}-G S D$ and for every $t>0$ the operators $e^{-t H}: L^{2}\left(\mathbf{R}^{d}, d x\right) \rightarrow L^{\infty}\left(\mathbf{R}^{d}\right)$ are bounded (i.e., the semigroup $\left\{e^{-t H}: t \geq 0\right\}$ is ultracontractive), then the semigroup $\left\{e^{-t H}: t \geq 0\right\}$ is $I S C$.

Proof. We again apply Lemma 2.1 for all $t>0$.

\section{INTRINSIC CONTRACTIVITY-TYPE PROPERTIES FOR JUMP-PARING LÉVY PROCESSES}

\subsection{Underlying Lévy processes and the corresponding non-local Schrödinger operators.} Let $\left(X_{t}\right)_{t \geq 0}$ be a symmetric Lévy process with values in $\mathbf{R}^{d}, d \geq 1$, with probability measure $\mathbf{P}^{x}$ of the process starting from $x \in \mathbf{R}^{d}$. We use the notation $\mathbf{E}^{x}$ for expectation with respect to $\mathbf{P}^{x}$. Recall that $\left(X_{t}\right)_{t \geq 0}$ is a Markov process with respect to its natural filtration, satisfying the strong Markov property and having càdlàg paths. It is determined by the characteristic function

$$
\mathbf{E}^{0}\left[e^{i \xi \cdot X_{t}}\right]=e^{-t \psi(\xi)}, \quad \xi \in \mathbf{R}^{d}, t>0
$$

with the characteristic exponent given by the Lévy-Khintchin formula

$$
\psi(\xi)=A \xi \cdot \xi+\int_{\mathbf{R}^{d}}(1-\cos (\xi \cdot z)) \nu(d z) .
$$

Here $A=\left(a_{i j}\right)_{1 \leq i, j \leq d}$ is a symmetric non-negative definite matrix, and $\nu$ is a symmetric Lévy measure on $\mathbf{R}^{d} \backslash\{0\}$, i.e., $\int_{\mathbf{R}^{d}}\left(1 \wedge|z|^{2}\right) \nu(d z)<\infty$ and $\nu(E)=\nu(-E)$, for every Borel set $E \subset$ $\mathbf{R}^{d} \backslash\{0\}$. For more details on Lévy processes we refer to [40, 20].

We will assume throughout that

$$
\nu\left(\mathbf{R}^{d} \backslash\{0\}\right)=\infty \quad \text { and } \quad \nu(d x)=\nu(x) d x, \quad \text { with } \quad \nu(x)>0 .
$$

For simplicity, we denote the density of the Lévy measure also by $\nu$ as it is the object we will use below. Note that the properties (3.2) jointly imply that $\left(X_{t}\right)_{t \geq 0}$ is a strong Feller process, or equivalently, there exist measurable transition probability densities $p(t, x, y)=p(t, 0, y-x)=$ : $p(t, y-x)$ with respect to Lebesgue measure such that $\mathbf{P}^{0}\left(X_{t} \in E\right)=\int_{E} p(t, x) d x$, for every Borel set $E \subset \mathbf{R}^{d}$ (see e.g. [40, Th. 27.7]). The transition probability densities $p_{D}(t, x, y)$ of the process killed upon exiting an open bounded set $D \subset \mathbf{R}^{d}$ are given by the Dynkin-Hunt formula

$$
p_{D}(t, x, y)=p(t, y-x)-\mathbf{E}^{x}\left[\tau_{D}<t ; p\left(t-\tau_{D}, y-X_{\tau_{D}}\right)\right], \quad x, y \in D,
$$

where $\tau_{D}=\inf \left\{t \geq 0: X_{t} \notin D\right\}$ is the first exit time of the process from $D$. The Green function is given by $G_{D}(x, y)=\int_{0}^{\infty} p_{D}(t, x, y) d t$, for all $x, y \in D$, and $G_{D}(x, y)=0$ if $x \notin D$ or $y \notin D$.

We will use the following class of Lévy processes (cf. [26]).

Definition 3.1 (Symmetric jump-paring Lévy processes). Let $\left(X_{t}\right)_{t \geq 0}$ be a Lévy process with Lévy-Khintchin exponent $\psi$ as in (3.1) -(3.2), satisfying the following conditions.

(A1) Lévy density: There exist a non-increasing profile function $g:(0, \infty) \rightarrow(0, \infty)$ and constants $C_{1}, C_{2}>0$ such that

$$
\nu(x) \asymp C_{1} g(|x|), \quad x \in \mathbf{R}^{d} \backslash\{0\},
$$


and

$$
\int_{\substack{|x-y|>1 \\|y|>1}} g(|x-y|) g(|y|) d y \leq C_{2} g(|x|), \quad|x| \geq 1 .
$$

(A2) Transition density: There exists $t_{\mathrm{b}}>0$ such that $\sup _{x \in \mathbf{R}^{d}} p\left(t_{\mathrm{b}}, x\right)=p\left(t_{\mathrm{b}}, 0\right)<\infty$.

(A3) Green function: For all $0<p<q<R \leq 1$ we have

$$
\sup _{x \in B(0, p)} \sup _{y \in B(0, q)^{c}} G_{B(0, R)}(x, y)<\infty .
$$

We call $\left(X_{t}\right)_{t \geq 0}$ satisfying the above conditions a symmetric jump-paring Lévy process and refer to the convolution condition in (3.4) as the jump-paring property.

The bound in (3.4) provides a control of the convolutions of $\nu$ with respect to large jumps and has a structural importance in defining the class of processes we consider. It says that the intensity of double large jumps of the process are dominated by the intensity of a single large jump. Let $\nu_{1}(x)=\nu(x) \mathbf{1}_{B(0,1)^{c}}(x)$. It is then seen iteratively that under (3.4) in fact

$$
\nu_{1}^{n *}(x) \leq C_{3}^{n-1} \nu_{1}(x), \quad|x| \geq 1, n \in \mathbf{N},
$$

holds, which means that every sequence of any finite length of large jumps of the process is dominated by single large jumps, which gives the name to the class of Lévy processes above.

The convolution condition (3.4) has been introduced in [25] and proved to be a strong tool in studying large-scale properties of non-local Schrödinger semigroups related to jump Lévy processes. Results obtained in [25] include sharp estimates on the ground state and upper estimates on the other eigenfunctions at infinity, and necessary and sufficient conditions for intrinsic ultracontractivity and its asymptotic version obtained via $L^{\infty}$-GSD and $L^{\infty}$-AGSD properties. Our present investigations complement this by focusing on further contractivity properties: $L^{p}$-GSD for $p \in(2, \infty)$, intrinsic supercontractivity and intrinsic hypercontractivity. Since below we often refer to [25], we note that under (3.1)-(3.2), our present conditions (A2)-(A3) coincide with Assumptions 2.2-2.3 there, while (A1) is a variant of Assumption 2.1. For simplicity, in the present paper this assumption is stated in terms of a profile function $g$, which gives some more regularity on the behaviour of $\nu$ around the origin; this is only a slight technical difference which has no impact on the results obtained here or in the referred paper. Recently, in [29] condition (3.4) has also been used to characterize the short-time behaviour of heat kernels for a large class of convolution semigroups. It can easily be checked that (A1) in fact implies the comparability condition [29, Lem. 1(a)]

$$
g(|x|) \asymp g(|x|+1), \quad|x| \geq 1 .
$$

Assumption (A2) is equivalent with $e^{-t_{\mathrm{b}} \psi} \in L^{1}\left(\mathbf{R}^{d}\right)$, for some $t_{\mathrm{b}}>0$. In this case $p\left(t_{\mathrm{b}}, x\right)$ can be obtained by the Fourier inversion formula, which extends to all $t \geq t_{\mathrm{b}}$ by the Markov property of $\left(X_{t}\right)_{t \geq 0}$. Further details on the existence and properties of transition probability densities for Lévy processes can be found in [30] and the references therein. We also note that in many cases of interest Assumption (A3) follows directly from space-time estimates of the densities $p(t, x)$. Indeed, if Assumption (A2) holds and for every $r>0$ there exists $C=C(r)$ such that $\sup _{|x| \geq r} p(t, x) \leq C t$, $t>0$, then (A3) results by standard estimates, see [26, Lem. 2.2] and [6, Prop. 2.3].

The generator $L$ of the process $\left(X_{t}\right)_{t>0}$ is uniquely determined by its Fourier symbol

$$
\widehat{L f}(\xi)=-\psi(\xi) \widehat{f}(\xi), \quad \xi \in \mathbf{R}^{d}, f \in D(L),
$$


with domain $D(L)=\left\{f \in L^{2}\left(\mathbf{R}^{d}\right): \psi \widehat{f} \in L^{2}\left(\mathbf{R}^{d}\right)\right\}$. The generator is a negative, non-local selfadjoint operator with core $C_{0}^{\infty}\left(\mathbf{R}^{d}\right)$, and

$L f(x)=\sum_{i, j=1}^{d} a_{i j} \frac{\partial^{2} f}{\partial x_{j} \partial x_{i}}(x)+\int_{\mathbf{R}^{d}}\left(f(x+y)-f(x)-y \cdot \nabla f(y) \mathbf{1}_{\{|y| \leq 1\}}\right) \nu(y) d y, \quad x \in \mathbf{R}^{d}, f \in C_{0}^{\infty}\left(\mathbf{R}^{d}\right)$.

There is a vast supply of Lévy processes satisfying Assumptions (A1)-(A3), including large subclasses of isotropic unimodal Lévy processes, subordinate Brownian motions, Lévy processes with non-degenerate Brownian components, symmetric stable-like processes, or processes with subexponentially or exponentially localized Lévy measures. In particular, it covers non-Gaussian isotropic stable and relativistic stable processes $\left(L=-\left(-\Delta+m^{2 / \alpha}\right)^{\alpha / 2}+m, \alpha \in(0,2), m \geq 0\right)$, jump-diffusions $\left(L=\Delta-(-\Delta)^{\alpha / 2}, \alpha \in(0,2)\right)$, geometric stable processes $\left(L=-\log \left(1+(-\Delta)^{\alpha / 2}\right)\right.$, $\alpha \in(0,2))$ and all of the examples discussed in detail in [25, Sect. 4].

We choose the class of potentials in a way which allows us to construct Feynman-Kac semigroups.

Definition 3.2 (X-Kato class). We say that the Borel function $V: \mathbf{R}^{d} \rightarrow \mathbf{R}$ called potential belongs to Kato-class $\mathcal{K}^{X}$ associated with the Lévy process $\left(X_{t}\right)_{t \geq 0}$ if it satisfies

$$
\lim _{t \downarrow 0} \sup _{x \in \mathbf{R}^{d}} \mathbf{E}^{x}\left[\int_{0}^{t}\left|V\left(X_{s}\right)\right| d s\right]=0 .
$$

Also, we say that $V$ is an $X$-Kato decomposable potential, whenever

$$
V=V_{+}-V_{-}, \quad \text { with } \quad V_{-} \in \mathcal{K}^{X} \quad \text { and } \quad V_{+} \in \mathcal{K}_{\text {loc }}^{X}
$$

where $V_{+}, V_{-}$denote the positive and negative parts of $V$, respectively, and where $V_{+} \in \mathcal{K}_{\text {loc }}^{X}$ means that $V_{+} 1_{B} \in \mathcal{K}^{X}$ for all compact sets $B \subset \mathbf{R}^{d}$.

For simplicity, we refer to $X$-Kato decomposable potentials as $X$-Kato class potentials. It is straightforward that $L_{\text {loc }}^{\infty}\left(\mathbf{R}^{d}\right) \subset \mathcal{K}_{\text {loc }}^{X}$, and by stochastic continuity of $\left(X_{t}\right)_{t \geq 0}$ also $\mathcal{K}_{\text {loc }}^{X} \subset L_{\text {loc }}^{1}\left(\mathbf{R}^{d}\right)$. Note that condition (3.7) allows local singularities of $V$. For specific processes $\left(X_{t}\right)_{t \geq 0}$ the definition of $X$-Kato class can be reformulated more explicitly in terms of the kernel $p(t, x)$ restricted to small $t$ and small $x$. It is shown in [14, Cor. 1.3] that (3.7) is equivalent with

$$
\lim _{t \rightarrow 0^{+}} \sup _{x \in \mathbf{R}^{d}} \int_{0}^{t} \int_{B(x, t)} p(s, x-y)|V(y)| d y d s=0 .
$$

In this section we consider confining potentials in the following sense.

(A4) Let $V$ be an $X$-Kato class potential such that $V(x) \rightarrow \infty$ as $|x| \rightarrow \infty$.

Next we define

$$
T_{t} f(x)=\mathbf{E}^{x}\left[e^{-\int_{0}^{t} V\left(X_{s}\right) d s} f\left(X_{t}\right)\right], \quad f \in L^{2}\left(\mathbf{R}^{d}\right), t>0 .
$$

Standard arguments based on Khasminskii's Lemma (see, e.g., [36, Lem. 3.37-3.38]) imply for an $X$-Kato class potential $V$ that there exist constants $C_{4}, C_{5}>0$ such that

$$
\sup _{x \in \mathbf{R}^{d}} \mathbf{E}^{x}\left[e^{-\int_{0}^{t} V\left(X_{s}\right) d s}\right] \leq \sup _{x \in \mathbf{R}^{d}} \mathbf{E}^{x}\left[e^{\int_{0}^{t} V_{-}\left(X_{s}\right) d s}\right] \leq C_{4} e^{C_{5} t}, \quad t>0 .
$$

Using the Markov property and stochastic continuity of the process it can be shown that $\left\{T_{t}: t \geq 0\right\}$ is a strongly continuous semigroup of symmetric operators on $L^{2}\left(\mathbf{R}^{d}\right)$, which we call the FeynmanKac semigroup associated with the process $\left(X_{t}\right)_{t \geq 0}$ and potential $V$. In particular, by the HilleYoshida theorem there exists a self-adjoint operator $H$, bounded from below, such that $e^{-t H}=T_{t}$. We call the operator $H$ a non-local Schrödinger operator based on the infinitesimal generator $L$ of 
the process $\left(X_{t}\right)_{t \geq 0}$. Since any $X$-Kato class potential is relatively form bounded with respect to $H_{0}=-L$ with relative bound less than 1 , the operator $H=H_{0}+V$ is also well-defined in form sense, see [12, Ch. 2].

The following are some basic properties of the operators $T_{t}$ needed below, for a proof see [25, Lem. 2.1].

Lemma 3.1. Let $\left(X_{t}\right)_{t \geq 0}$ be a symmetric Lévy process with Lévy-Khintchin exponent satisfying (3.1) - (3.2) such that Assumption (A2) holds, and let $V$ be an $X$-Kato class potential. Then the following properties hold:

(1) For all $t>0$, every $T_{t}$ is a bounded operator on every $L^{p}\left(\mathbf{R}^{d}, d x\right)$ space, $1 \leq p \leq \infty$. The operators $T_{t}: L^{p}\left(\mathbf{R}^{d}, d x\right) \rightarrow L^{p}\left(\mathbf{R}^{d}, d x\right)$ for $1 \leq p \leq \infty, t>0$, and $T_{t}: L^{p}\left(\mathbf{R}^{d}, d x\right) \rightarrow$ $L^{\infty}\left(\mathbf{R}^{d}, d x\right)$ for $1<p \leq \infty, t \geq t_{\mathrm{b}}$, and $T_{t}: L^{1}\left(\mathbf{R}^{d}, d x\right) \rightarrow L^{\infty}\left(\mathbf{R}^{d}, d x\right)$ for $t \geq 2 t_{\mathrm{b}}$ are bounded.

(2) For all $t>0$ the operators $T_{t}: L^{2}\left(\mathbf{R}^{d}, d x\right) \rightarrow L^{2}\left(\mathbf{R}^{d}, d x\right)$ are compact.

(3) For all $t>0$ and $f \in L^{\infty}\left(\mathbf{R}^{d}, d x\right), T_{t} f$ is a bounded continuous function.

(4) For all $t>0$ the operators $T_{t}$ are positivity improving.

Note that we do not assume that $p(t, x)$ is bounded for all $t>0$, and thus in general the operators $T_{t}: L^{2}\left(\mathbf{R}^{d}, d x\right) \rightarrow L^{\infty}\left(\mathbf{R}^{d}, d x\right)$ need not be bounded for $t<t_{\mathrm{b}}$. Also, note that from the above it follows that the semigroup $\left\{T_{t}: t \geq 0\right\}$ satisfies the basic regularity conditions (a)-(d) in Section 2.

The following upper bound on the ground state of $H$ at infinity obtained in [25, Cor. 2.2] will be an important ingredient below, guaranteeing that for the class of processes and potentials considered in this section we have $\varphi_{0} \in L^{1}\left(\mathbf{R}^{d}, d x\right)$. For $r>0$ denote $V_{r}^{*}(x):=\sup _{y \in B(x, r)} V(y), x \in \mathbf{R}^{d}$.

Proposition 3.1. Let $\left(X_{t}\right)_{t \geq 0}$ be a symmetric Lévy process determined by (3.1)-(3.2) with defining parameters $A$ and $\nu$ such that Assumptions (A1)-(A3) hold, and let $V$ be a potential satisfying Assumption (A4). Then for every $r \in(0,1]$ there exists $C_{6}>0$ and $R>0$ such that

$$
\frac{1}{C_{6}} \frac{\nu(x)}{V_{r}^{*}(x)} \leq \varphi_{0}(x) \leq C_{6} \nu(x), \quad|x| \geq R .
$$

3.2. Necessary and sufficient conditions for $L^{p}$-ground state domination. We begin with the following result providing sufficient conditions for $L^{p}$-GSD and $L^{p}$-AGSD. It directly follows from the fact that $L^{\infty}$-GSD and $L^{\infty}$-AGSD imply $L^{p}$-GSD and $L^{p}$-AGSD for any $p \in(2, \infty)$, respectively.

Proposition 3.2. (Sufficient conditions for $L^{p}$-GSD and $L^{p}$-AGSD) Let $\left(X_{t}\right)_{t \geq 0}$ be a symmetric Lévy process determined by (3.1) -(3.2) with defining parameters $A$ and $\nu$ such that Assumptions (A1)-(A3) hold, and let $V$ be a potential satisfying Assumption (A4). Then the following hold:

(i) If there exist constants $C_{7}>0$ and $R>0$ such that

$$
\frac{V(x)}{|\log \nu(x)|} \geq C_{7}, \quad \text { for every }|x| \geq R,
$$

then for every $p \in(2, \infty)$ the semigroup $\left\{T_{t}: t \geq 0\right\}$ is $\left(t_{0}, L^{p}\right)$-AGSD with $t_{0}=4 / C_{7}$.

(ii) If

$$
\lim _{|x| \rightarrow \infty} \frac{V(x)}{|\log \nu(x)|}=\infty
$$

then for every $p \in(2, \infty)$ the semigroup $\left\{T_{t}: t \geq 0\right\}$ is $L^{p}$-GSD. 
Proof. By [25, Ths. 2.6-2.7] the conditions in (i) and (ii) imply $\left(t_{0}, L^{\infty}\right)$-AGSD (with $t_{0}=4 / C_{7}$ ) and $L^{\infty}$-GSD, respectively. Moreover, as seen above, $L^{\infty}\left(\mathbf{R}^{d}, \mu\right) \subset L^{p}\left(\mathbf{R}^{d}, \mu\right)$, for any $p \in(2, \infty)$.

It is tempting to expect that $L^{\infty}{ }_{-}$GSD and $L^{\infty}$-AGSD are much stronger than $L^{p_{-} \mathrm{GSD}}$ and $L^{p_{-}}$ AGSD for $p<\infty$, and so the above proposition may seem not to give a sharp result. However, this intuition is false. Below we prove that for a large class of confining potentials $L^{\infty}$-GSD and $L^{\infty}$-AGSD are equivalent with $L^{p}$-GSD and $L^{p}$-AGSD, for every $p \in(2, \infty)$.

The following theorem is our main result in this section, giving direct necessary conditions for $L^{p}$-GSD and $L^{p}$-AGSD. This observation actually leads to a full characterization of these properties in terms of the Lévy density $\nu$ and the potential $V$.

Theorem 3.1. (Necessary conditions for $L^{p}$-GSD and $L^{p}$-AGSD) Let $\left(X_{t}\right)_{t \geq 0}$ be a symmetric Lévy process determined by (3.1)-(3.2) with defining parameters $A$ and $\nu$ such that Assumptions (A1)-(A3) hold, and let $V$ be a potential satisfying (A4). Then we have:

(i) If for some $p \in(2, \infty)$ the semigroup $\left\{T_{t}: t \geq 0\right\}$ is $\left(t_{0}, L^{p}\right)$-AGSD, then for every $r \in(0,1)$ and $\varepsilon \in\left(0,(p-2) /\left(p t_{0}\right)\right)$ there exists $R>0$ such that

$$
\frac{V_{r}^{*}(x)}{|\log \nu(x)|} \geq \frac{p-2}{p t_{0}}-\varepsilon, \quad \text { for every }|x| \geq R .
$$

(ii) If for some $p \in(2, \infty)$ the semigroup $\left\{T_{t}: t \geq 0\right\}$ is $L^{p}$-GSD, then for every $r \in(0,1)$

$$
\lim _{|x| \rightarrow \infty} \frac{V_{r}^{*}(x)}{|\log \nu(x)|}=\infty .
$$

Proof. (i) By the definition of $\left(t_{0}, L^{p}\right)$-AGSD, for every $x \in \mathbf{R}^{d}$ and $r \in(0,1]$ we have

$$
\int_{|x-y|<r / 2} \frac{\left|T_{t_{0}} \mathbf{1}(y)\right|^{p}}{\varphi_{0}^{p-2}(y)} d y \leq \int_{\mathbf{R}^{d}}\left|\frac{T_{t_{0}} \mathbf{1}(y)}{\varphi_{0}(y)}\right|^{p} \varphi_{0}^{2}(y) d y<\infty
$$

We first estimate the term under the integral at the left hand side above for $y \in B(x, r / 2)$ :

$$
\begin{aligned}
T_{t_{0}} \mathbf{1}(y) & \geq \mathbf{E}^{y}\left[e^{-\int_{0}^{t_{0}} V\left(X_{s}\right) d s} ; t_{0}<\tau_{B(y, r / 2)}\right] \\
& \geq e^{-t_{0} \sup _{z \in B(y, r / 2)} V(z)} \mathbf{P}^{y}\left(t_{0}<\tau_{B(y, r / 2)}\right) \geq e^{-t_{0} V_{r}^{*}(x)} \mathbf{P}^{0}\left(t_{0}<\tau_{B(0, r / 2)}\right) .
\end{aligned}
$$

By Proposition 3.1 and (3.5) there exists $R \geq 1$ such that

$$
\varphi_{0}(y) \leq c_{1} \nu(y) \leq c_{2} \nu(x) \quad \text { and } \quad \nu(x) \leq 1, \quad \text { whenever }|x|>R \text { and }|x-y| \leq r / 2 .
$$

Thus by (3.10) there exist constants $c_{3}=c_{3}\left(p, t_{0}\right)$ and $c_{4}=c_{4}\left(p, t_{0}, r\right)$ such that for $|x|>R$

$$
e^{-t_{0} V_{r}^{*}(x)} \leq c_{3} \frac{\nu(x)^{\frac{p-2}{p}}}{(|B(0, r / 2)|)^{1 / p} \mathbf{P}^{0}\left(t_{0}<\tau_{B(0, r / 2)}\right)}
$$

and

$$
\frac{V_{r}^{*}(x)}{|\log \nu(x)|} \geq \frac{p-2}{p t_{0}}-\frac{c_{4}}{|\log \nu(x)|} .
$$

Since $|\log \nu(x)| \rightarrow \infty$ as $|x| \rightarrow \infty$, this completes the proof of (i).

To show (ii), it suffices to observe that $L^{p}$-GSD implies $\left(t_{0}, L^{p}\right)$-AGSD for any $t_{0}>0$. By following through the above argument, we obtain

$$
\liminf _{|x| \rightarrow \infty} \frac{V_{r}^{*}(x)}{|\log \nu(x)|} \geq \frac{p-2}{p t_{0}} .
$$

Letting $t_{0} \rightarrow 0^{+}$, the claim follows. 
It follows from Theorems 2.1 2.2 that under condition (2.3) Theorem 3.1 above also gives necessary conditions for IHC and ISC. Next we show that in the framework of this section the same holds without requiring (2.3).

Corollary 3.1. (Necessary and sufficient conditions for ISC and IHC) Let $\left(X_{t}\right)_{t \geq 0}$ be a symmetric Lévy process determined by (3.1)-(3.2) with defining parameters $A$ and $\nu$ such that Assumptions (A1)-(A3) hold, and let $V$ be a potential satisfying Assumption (A4). Then we have:

(i) The condition in Proposition 3.2 (i) is sufficient for IHC. Conversely, if IHC holds, then for every $p \in(3, \infty)$ and $r \in(0,1)$ there exist $t_{p}>0$ and $R>0$ such that

$$
\frac{V_{r}^{*}(x)}{|\log \nu(x)|} \geq \frac{p-3}{2 p t_{0}}, \quad \text { for every }|x| \geq R
$$

(ii) The condition in Proposition 3.2 (ii) is sufficient for ISC. Conversely, if ISC holds, then for every $r \in(0,1)$

$$
\lim _{|x| \rightarrow \infty} \frac{V_{r}^{*}(x)}{|\log \nu(x)|}=\infty
$$

Proof. We only need to consider the necessary conditions. Similarly as before, it is enough to justify that in (i). Suppose IHC holds and fix $r \in(0,1]$. By (2.3) and the Remark 2.1(3) we have that for every $p \in(3, \infty)$ there exists $t_{p}>0$ such that $\frac{e^{-2 t_{p} H} \varphi_{0}^{\frac{1}{p}}}{\varphi_{0}} \in L^{p}\left(\mathbf{R}^{d}, \mu\right)$. Thus by using both the lower and upper bounds in Proposition 3.1 and by following the argument in the proof of Theorem 3.1 (i), we get that there exists $R>0$ such that for $|x|>R$

$$
\frac{e^{-t_{p} V_{r}^{*}(x)}}{\left(V_{r}^{*}(x)\right)^{1 / p}} \leq c_{1} \frac{\nu(x)^{\frac{p-3}{p}}}{(|B(0, r / 2)|)^{1 / p} \mathbf{P}^{0}\left(t_{p}<\tau_{B(0, r / 2)}\right)}
$$

and

$$
\frac{V_{r}^{*}(x)+(1 / p) \log V_{r}^{*}(x)}{|\log \nu(x)|} \geq \frac{p-3}{p t_{p}}-\frac{c_{2}}{|\log \nu(x)|},
$$

with some constants $c_{1}, c_{2}>0$. We see that by increasing $R>0$ if necessary, we get the claimed inequality.

Then the following characterization is a direct corollary of Proposition 3.2 and Theorem 3.1 .

Theorem 3.2. (Characterization of $L^{p}$-GSD and $L^{p}$-AGSD) Let $\left(X_{t}\right)_{t \geq 0}$ be a symmetric Lévy process determined by (3.1)-(3.2) with defining parameters $A$ and $\nu$ such that (A1)-(A3) hold, and let $V$ satisfy Assumption (A4). Moreover, suppose that the potential $V$ satisfies at least one of the following additional assumptions:

(A5) There exist $r \in(0,1)$ and $R>0$ such that $V_{r}^{*}(x) \asymp V(x)$, for $|x|>R$.

(A6) There exist a non-decreasing function $f$ and $R>0$ such that $V(x) \asymp f(|x|)$, for $|x|>R$.

The following hold:

(i) The semigroup $\left\{T_{t}: t \geq 0\right\}$ is $L^{p}$-GSD for every $p \in(2, \infty)$ if and only if

$$
\lim _{|x| \rightarrow \infty} \frac{V(x)}{|\log \nu(x)|}=\infty .
$$

(ii) The semigroup $\left\{T_{t}: t \geq 0\right\}$ is $L^{p}$-AGSD for every $p \in(2, \infty)$ if and only if there exist constants $C>0$ and $R>0$ such that

$$
\frac{V(x)}{|\log \nu(x)|} \geq C, \quad \text { for every }|x| \geq R .
$$


Proof. Under (A5) the result follows directly from Proposition 3.2 and Theorem 3.1 above. Suppose now that (A6) holds. It suffices to prove that the conditions on $V$ and $\nu$ in (i) and (ii) are in fact necessary for $L^{p}$-GSD and $L^{p}$-AGSD, respectively.

Consider first (ii). If $\left(t_{0}, L^{p}\right)$-AGSD holds for some $p \in(2, \infty)$ and $t_{0}>0$, then by a similar argument as in the proof of Theorem 3.1 (i) and by (A6), there exist constants $R>0, c_{1}=c_{1}\left(p, t_{0}\right)$ and $c_{2}>0$ (independent of $t_{0}$ and $p$ ) such that

$$
c_{2} V(x+x /|x|) \geq \frac{p-2}{p t_{0}}|\log \nu(x)|-c_{1}, \quad \text { for every }|x| \geq R,
$$

which is equivalent with

$$
V(x) \geq \frac{p-2}{c_{2} p t_{0}}|\log \nu(x-x /|x|)|-\frac{c_{1}}{c_{2}}, \quad \text { for every }|x| \geq R+1 .
$$

By (A1) and (3.5) there exists a constant $c_{3}>0$ (also independent of $t_{0}$ and $p$ ) such that

$$
|\log \nu(x-x /|x|)| \geq|\log \nu(x)|-c_{3}, \quad \text { for sufficiently large }|x| .
$$

Since $|\log \nu(x)| \rightarrow \infty$ as $|x| \rightarrow \infty$, the assertion (ii) follows. To get (i), it is enough to observe that

$$
\liminf _{|x| \rightarrow \infty} \frac{V(x)}{|\log \nu(x)|} \geq \frac{p-2}{c_{2} p t_{0}}
$$

and take the limit $t_{0} \rightarrow 0^{+}$.

3.3. Equivalence of intrinsic contractivity properties for non-local Schrödinger operators. The next two results summarize our investigations in the present paper. They say that for a large class of potentials (at least those satisfying Assumption (A5) or (A6) above) ISC is equivalent to intrinsic ultracontractivity (IUC), and IHS is equivalent to asymptotic intrinsic ultracontractivity (AIUC). This surprising fact has not been noted before, and it shows a different behaviour from the classical case. Indeed, for Schrödinger operators $H=-\Delta+V$ all these contractivity properties are different, even when the potentials are quite regular (see [11, p.336] and the references therein). First we recall the following definitions from [11] and [25]. In what follows, $\left\{\widetilde{T}_{t}: t \geq 0\right\}$ will denote the ground state-transformed semigroup to $\left\{T_{t}: t \geq 0\right\}$.

\section{Definition 3.3. (Intrinsic ultracontractivity-type properties)}

(i) The semigroup $\left\{\widetilde{T}_{t}: t \geq 0\right\}$ is ultracontractive if for every $t>0$ the operators $\widetilde{T}_{t}$ are bounded from $L^{2}\left(\mathbf{R}^{d}, \mu\right)$ to $L^{\infty}\left(\mathbf{R}^{d}\right)$.

(ii) The semigroup $\left\{T_{t}: t \geq 0\right\}$ is called intrinsically ultracontractive (abbreviated as IUC) if the semigroup $\left\{\widetilde{T}_{t}: t \geq 0\right\}$ is ultracontractive.

(iii) The semigroup $\left\{\widetilde{T}_{t}: t \geq 0\right\}$ is asymptotically ultracontractive if there exists $t_{0}>0$ such that for every $t \geq t_{0}$ the operators $\widetilde{T}_{t}$ are bounded from $L^{2}\left(\mathbf{R}^{d}, \mu\right)$ to $L^{\infty}\left(\mathbf{R}^{d}\right)$.

(iv) The semigroup $\left\{T_{t}: t \geq 0\right\}$ is asymptotically intrinsically ultracontractive (abbreviated as AIUC) if the semigroup $\left\{\widetilde{T}_{t}: t \geq 0\right\}$ is asymptotically ultracontractive.

The following results are direct consequences of the above results and [25, Th. 2.5, Cor. 2.3].

Corollary 3.2. (Equivalence of intrinsic contractivity properties) Let $\left(X_{t}\right)_{t \geq 0}$ be a symmetric Lévy process determined by (3.1)-(3.2) with defining parameters $A$ and $\nu$ such that Assumptions (A1)-(A3) hold, and let $V$ be a potential satisfying Assumption (A4) and at least one of the additional Assumptions (A5) or (A6). Moreover, suppose that the transition densities $p(t, \cdot)$ are bounded for all $t>0$. Then the following properties are equivalent:

(i) $\lim _{|x| \rightarrow \infty} \frac{V(x)}{|\log \nu(x)|}=\infty$. 
(ii) The semigroup $\left\{T_{t}: t \geq 0\right\}$ is $L^{p}$-GSD for every $p \in(2, \infty]$.

(iii) The semigroup $\left\{T_{t}: t \geq 0\right\}$ is ISC.

(iv) The semigroup $\left\{T_{t}: t \geq 0\right\}$ is IUC.

Corollary 3.3. (Equivalence of asymptotic intrinsic contractivity properties) Let $\left(X_{t}\right)_{t \geq 0}$ be a symmetric Lévy process determined by (3.1)-(3.2) with defining parameters $A$ and $\nu$ such that Assumptions (A1)-(A3) hold, and let $V$ be a potential satisfying Assumption (A4) and at least one of the additional Assumptions (A5) or (A6). Then the following properties are equivalent:

(i) There exist $C, R>0$ such that $\frac{V(x)}{|\log \nu(x)|} \geq C$, for every $|x| \geq R$.

(ii) The semigroup $\left\{T_{t}: t \geq 0\right\}$ is $L^{p}$-AGSD for every $p \in(2, \infty]$.

(iii) The semigroup $\left\{T_{t}: t \geq 0\right\}$ is IHC.

(iv) The semigroup $\left\{T_{t}: t \geq 0\right\}$ is AIUC.

We close this section by noting that for our class of jump-paring Lévy processes and for confining potentials satisfying (A5) or (A6) the function $|\log \nu(x)|$ in fact determines the borderline growth of the potential for intrinsic contractivity properties listed in Corollaries $3.2[3.3$ above.

\section{EXAMPLES}

To illustrate these results, we assume for simplicity that $\left(X_{t}\right)_{t \geq 0}$ is a symmetric Lévy process with characteristic exponent $\psi$ satisfying (3.1)-(3.2), and parameters $A=a \operatorname{Id}$ for $a \geq 0$ and $\nu$ satisfying (3.3) for the profile function $g$, such that $g(r)=r^{-d-\alpha}$ with some $\alpha \in(0,2)$, for all $r \in(0,1]$. Assumptions (A1)-(A3) for the processes discussed in Examples 4.1 and 4.3 below have been verified in [25, Sect. 4]; for Example 4.2 they can be checked similarly, and we leave the details to the reader.

Example 4.1. (Processes with polynomially suppressed large jumps) Let $g(r)=r^{-d-\gamma}$ with $\gamma>0$, for $r \geq 1$. This class includes

(i) rotationally invariant $\alpha$-stable process $(\gamma=\alpha)$

(ii) mixture of $i \geq 2$ rotation invariant $\alpha_{i}$-stable processes with indices $\alpha_{i} \in(0,2]\left(\gamma=\min \alpha_{i}\right)$

(iii) layered $\alpha$-stable process $(\gamma>2)$.

Let $V$ be an $X$-Kato class potential such that

$$
V_{+}(x) \asymp f(|x|), \quad \text { with } f(r)=(1+r)^{\delta_{1}}[\log (2+r)]^{\delta_{2}}\left[\log (2+\log (2+r)]^{\delta_{3}} \text { for } \delta_{1}, \delta_{2}, \delta_{3} \in \mathbf{R} .\right.
$$

Then the four equivalent conditions in Corollary 3.2 hold if and only if
(1) $\delta_{1}>0, \delta_{2} \in \mathbf{R}, \delta_{3} \in \mathbf{R} \quad$ or
(2) $\delta_{1}=0, \delta_{2}>1, \delta_{3} \in \mathbf{R} \quad$ or
(3) $\delta_{1}=0, \delta_{2}=1, \delta_{3}>0$.

Also, the four equivalent conditions in Corollary 3.3 hold if and only if
(1) $\delta_{1}>0, \delta_{2} \in \mathbf{R}, \delta_{3} \in \mathbf{R} \quad$ or
(2) $\delta_{1}=0, \delta_{2} \geq 1, \delta_{3} \in \mathbf{R} \quad$ or
(3) $\delta_{1}=0, \delta_{2}=1, \delta_{3} \geq 0$.

Example 4.2. (Processes with stretched-exponentially suppressed large jumps) Let $g(r)=$

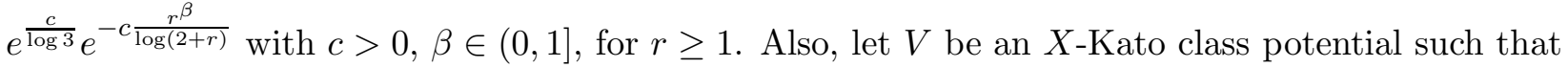
$V_{+}(x) \asymp f(|x|)$, with $f(r)=(1+r)^{\delta_{1}}[\log (2+r)]^{\delta_{2}}$ for $\delta_{1}, \delta_{2} \in \mathbf{R}$.

Then the four equivalent conditions in Corollary 3.2 hold if and only if
(1) $\delta_{1}>1, \delta_{2} \in \mathbf{R}$
or
(2) $\delta_{1}=1, \delta_{2}>-1$.

Moreover, the four equivalent conditions in Corollary 3.3 hold if and only if
(1) $\delta_{1}>1, \delta_{2} \in \mathbf{R}$
or
(2) $\delta_{1}=1, \delta_{2} \geq-1$. 
Example 4.3. (Processes with exponentially suppressed large jumps) Let $g(r)=e^{c} e^{-c r} r^{-\gamma}$ with $c>0, \gamma>(d+1) / 2$, for $r \geq 1$. This class includes

(i) relativistic $\alpha$-stable process $\left(c=m^{1 / \alpha}, \gamma=(d+\alpha+1) / 2, m>0\right)$,

(ii) (exponentially) tempered $\alpha$-stable process $(c>0, \gamma=d+\alpha)$.

Let $V$ be an $X$-Kato class potential such that

$$
V_{+}(x) \asymp f(|x|), \quad \text { with } f(r)=(1+r)^{\delta_{1}}[\log (2+r)]^{\delta_{2}} \text { for } \delta_{1}, \delta_{2} \in \mathbf{R} .
$$

Then the four equivalent conditions in Corollary 3.2 hold if and only if

$$
\text { (1) } \delta_{1}>1, \delta_{2} \in \mathbf{R} \quad \text { or } \quad \text { (2) } \delta_{1}=1, \delta_{2}>0 \text {. }
$$

Moreover, the four equivalent conditions in Corollary 3.3 hold if and only if

$$
\text { (1) } \delta_{1}>1, \delta_{2} \in \mathbf{R} \quad \text { or } \quad \text { (2) } \delta_{1}=1, \delta_{2} \geq 0
$$

Example 4.4. (Brownian motion) Consider the Schrödinger operator $H=-\Delta+V$ with $V(x)=$ $|x|^{\delta_{1}}\left(\log (2+|x|)^{\delta_{2}}\right.$, for $\delta_{1}, \delta_{2} \geq 0$, and denote by $\left(B_{t}\right)_{t \geq 0}$ Brownian motion running at twice the usual speed. As said before, the corresponding Schrödinger semigroup $\left\{e^{-t H}: t \geq 0\right\}$ is ISC but not IUC if $\delta_{1}=2$ and $0<\delta_{2} \leq 2$. Note that $C_{2, \infty, t}=\lim _{p \rightarrow \infty} C_{2, p, t}=\infty$, for every $t>0$, where $C_{2, p, t}$ is the norm of the operator $e^{-t \widetilde{H}}$ from $L^{2}\left(\mathbf{R}^{d}, \mu\right)$ to $L^{p}\left(\mathbf{R}^{d}, \mu\right), p \in(2, \infty]$, which shows a different behaviour from the non-local Schrödinger operators studied in this paper (cf. Corollary 3.2). Indeed, from the proof of Corollary 3.1 it can be seen that under Assumption (A5) or (A6) the finiteness of $C_{2, p, t}$ for some $p \in(2, \infty)$ in fact guarantees that $C_{2, \infty, t}<\infty$. Moreover, recall that when $\delta_{1}=2$ and $\delta_{2}=0$, then $\left\{e^{-t H}: t \geq 0\right\}$ is IHC but not ISC. This example also shows that for classical Schrödinger operators IHC is not equivalent with AIUC, unlike for non-local Schrödinger operators (cf. Corollary [3.3). Using that, see [37, eq.(2.1)],

$$
\varphi_{0}(x) \asymp e^{-\frac{|x|^{2}}{2}} \quad \text { and } \quad e^{-t H} \mathbf{1}(x)=\mathbf{E}^{x}\left[e^{-\int_{0}^{t}\left|B_{s}\right|^{2} d s}\right] \asymp e^{-\frac{|x|^{2}}{2 \operatorname{coth}(2 t)}}, \quad x \in \mathbf{R}^{d}, t>0,
$$

we see that for every $p \in(2, \infty)$ there exists $t_{p}>0$ such that for all $t \geq t_{p}$ the operator $e^{-t H}$ is $L^{p}$-GSD. On the other hand, there is no $t>0$ for which $e^{-t H}$ is $L^{\infty}$-GSD.

\section{REFERENCES}

[1] L. Acuña Valverde, R. Bañuelos: Heat content and small time asymptotics for Schrödinger Operators on $\mathbf{R}^{d}$, Potential Analysis 42 (2), 457-482, 2015.

[2] D. Bakry, I. Gentil, M. Ledoux: Analysis and Geometry of Markov Diffusion Operators, Springer, 2013

[3] Y.A. Butko, O.G. Smolyanov, R.L. Schilling: Hamiltonian Feynman-Kac and Feynman formulae for dynamics of particles with position-dependent mass, Int. J. Theor. Physics 50 (2011), 2009-2018.

[4] R. Bañuelos: Intrinsic ultracontractivity and eigenfunction estimates for Schrödinger operators, J. Funct. Anal. 100, 1991, 181-206.

[5] R. Bañuelos, S. Yildirim: Heat trace of non-local operators, J. Lond. Math. Soc. 87 (2013), 304-318.

[6] K. Bogdan, T. Kumagai, M. Kwaśnicki: Boundary Harnack inequality for Markov processes with jumps, Trans. Amer. Math. Soc. 367(1), 2015, 477-517.

[7] R. Carmona: Pointwise bounds for Schrödinger eigenstates, Commun. Math. Phys. 62, 1978, 65-92.

[8] R. Carmona, W.C. Masters, B. Simon: Relativistic Schrödinger operators: asymptotic behaviour of the eigenfunctions, J. Funct. Anal. 91, 1990, 117-142.

[9] X. Chen, J. Wang: Intrinsic contractivity properties of Feynman-Kac semigroups for symmetric jump processes with infinite range jumps, Frontiers of Mathematics in China 10 (4), 753-776 (2015).

[10] E.B. Davies: Heat Kernels and Spectral Theory, Cambridge University Press, 1990

[11] E.B. Davies, B. Simon: Ultracontractivity and the heat kernel for Schrödinger operators and Dirichlet Laplacians, J. Funct. Anal. 59 (1984) 335-395.

[12] M. Demuth, J. A. van Casteren: Stochastic Spectral Theory for Self-adjoint Feller Operators. A Functional Analysis Approach, Birkhäuser, Basel, 2000. 
[13] R. Frank, E. Lenzmann, L. Silvestre: Uniqueness of radial solutions for the fractional Laplacian, Commun. Pure Appl. Math., to appear.

[14] T. Grzywny, K. Szczypkowski: Kato classes for Lévy processes, preprint, 2015, available at arXiv:1503.05747

[15] M. Hirokawa, F. Hiroshima, J. Lörinczi: Spin-boson model through a Poisson-driven stochastic process, Math. Zeitschrift 277, 1165-1198, 2014

[16] F. Hiroshima, T. Ichinose, J. Lörinczi: Probabilistic representation and fall-off of bound states of relativistic Schrödinger operators with spin 1/2, Publ. Res. Inst. Math. Sci. 49, 2013, 189-214

[17] F. Hiroshima, J. Lörinczi: Functional integral representation of the Pauli-Fierz model with spin $1 / 2$, J. Funct. Anal. 254, 2127-2185, 2008

[18] F. Hiroshima, J. Lörinczi: Lieb-Thirring bound for Schrödinger operators with Bernstein functions of the Laplacian, Commun. Stoch. Anal. 6, 589-602, 2012

[19] F. Hiroshima, J. Lörinczi, T. Takaesu: A probabilistic representation of the ground state expectation of fractional powers of the boson number operator, J. Math. Anal. Appl. 395, 437-447, 2012

[20] N. Jacob: Pseudo-Differential Operators and Markov Processes: Markov Processes and Applications, vols. 1-3, Imperial College Press, 2003-2005.

[21] K. Kaleta, Spectral gap lower bound for the one-dimensional fractional Schrödinger operator in the interval, Studia Math. 209, 2012, 267-287

[22] K. Kaleta, T. Kulczycki: Intrinsic ultracontractivity for Schrödinger operators based on fractional Laplacians, Potential Anal. 33 (4), 2010, 313-339.

[23] K. Kaleta, M. Kwaśnicki, J. Małecki: One-dimensional quasi-relativistic particle in the box, Rev. Math. Phys. 25, 1350014,2013

[24] K. Kaleta, J. Lőrinczi: Fractional P( $\phi)_{1}$-processes and Gibbs measures, Stoch. Proc. Appl. 122, $2012,3580-3617$.

[25] K. Kaleta, J. Lörinczi: Pointwise eigenfunction estimates and intrinsic ultracontractivity-type properties of Feynman-Kac semigroups for a class of Lévy processes, Ann. Probab. 43 (3), 2015, 1350-1398.

[26] K. Kaleta, J. Lörinczi: Fall-off of eigenfunctions for non-local Schrödinger operators with decaying potentials, preprint 2015, available at arXiv:1503.03508

[27] K. Kaleta, J. Lörinczi: Transition in the decay rates of stationary distributions of Lévy motion in an energy landscape, preprint 2015, available at arXiv:1507.01746

[28] K. Kaleta, J. Lörinczi: Typical long time behaviour of ground state-transformed jump processes, preprint 2015.

[29] K. Kaleta, P. Sztonyk: Small time sharp bounds for kernels of convolution semigroups, J. Anal. Math, 2015, to appear, available at arXiv:1403.0912

[30] V. Knopova, R.L. Schilling: A note on the existence of transition probability densities for Lévy processes, To appear in Forum Math., arXiv:1003.1419.

[31] T. Kulczycki: Gradient estimates of q-harmonic functions of fractional Schrödinger operator, Potential Anal. 39 (2013), no. 1, 69-98.

[32] T. Kulczycki, M. Kwaśnicki, J. Małecki, A. Stós: Spectral properties of the Cauchy process on half-line and interval, Proc. London Math. Soc. 101, 589-622, 2010.

[33] M. Kwaśnicki: Eigenvalues of the fractional Laplace operator in the interval, J. Funct. Anal. 262, 2379-2402, 2012

[34] E.H. Lieb, R. Seiringer, The Stability of Matter in Quantum Mechanics, Cambridge University Press, 2009.

[35] J. Lörinczi, J. Małecki: Spectral properties of the massless relativistic harmonic oscillator, J. Diff. Equations 253 (2012), p. 2846-2871.

[36] J. Lörinczi, F. Hiroshima, V. Betz: Feynman-Kac-Type Theorems and Gibbs Measures on Path Space. With Applications to Rigorous Quantum Field Theory, Walter de Gruyter, 2011.

[37] R. Mansuy, M. Yor: Aspects of Brownian Motion, Springer, 2008

[38] E. Nelson: A quartic interaction in two dimensions, in: Mathematical Theory of Elementary Particles, R. Goodman, I. Segal (eds.), MIT Press, 1966, pp. 69-73

[39] M. Reed, B. Simon: Methods of Modern Mathematical Physics, vol. IV, Academic Press, 1980.

[40] K.I. Sato: Lévy Processes and Infinitely Divisible Distributions, Cambridge University Press, 1999.

[41] B. Simon: Schrödinger semigroups, Bull. Amer. Math. Soc. 7 (3), 1982, 447-526.

Faculty of Pure and Applied Mathematics, Wroceaw University of Technology, Wyb. Wyspiańskiego 27, 50-370 WrocŁaW, Poland

E-mail address: kamil.kaleta@pwr.edu.pl, mateusz.kwasnicki@pwr.edu.pl

Department of Mathematics, Loughborough University, Loughborough LE11 3TU, United Kingdom

E-mail address: J.Lorinczi@lboro.ac.uk 